involve any of the causes mentioned above. In such systems, chaotic behaviour appears without requiring the presence of additional noise sources or stochastic processes.

\section{A Unifying Principle}

In physics, one usually looks for unifying principles, as for example, the Laplace equation that makes a linear theory applicable to many fields. It is remarkable that the nonlinear effect in all of the phenomena listed above can be described in terms of simple models that lead to chaotic behaviour. On a microscopic level, the origin of chaos lies in the sensitive dependence on initial conditions, and the assertion of an inability to make measurements that are more precise than those implied by the uncertainity principle.

What then is the attribute which initiates a sensitive dependence on initial conditions? We have seen that integrability makes the orbits of a dynamical system lie on tori in phase space. We also know that continuous symmetry in a problem leads to the existence of an integral; when symmetry is broken, the system loses the integral associated with the symmetry and chaotic behaviour sets in. The detailed mechanism of symmetry breaking may differ from one case to another, but the gross features can be described in terms of chaos. It is the breaking of rotational symmetry in 2, 3 or 4 dimensions that is one of the common characteristics in many chaotic problems cited above.

The motion of a hydrogen atom in a magnetic field [5] is a simple problem which illustrates all of these properties. Furthermore, it is not a contrived model but a truly physical system where it is possible to compare theoretical calculations with experimental observations. The transitions from order to chaos depend on a single parameter, namely the magnetic field strength, which can be experimentally adjusted. The Hamiltonian (where $y$ is related to the strength of the magnetic field)

$$
\begin{aligned}
H & =p^{2} / 2-1 / r+y L x / 2 \\
& +y^{2}\left(x^{2}+y^{2}\right) / 8=E
\end{aligned}
$$

shows the transition from a fully spherical symmetry to cylindrical symmetry as the magnetic field is turned on. Upon introducing semi-parabolic coordinates, one obtains the Hamiltonian

$H_{\mathrm{o}}=p_{u}^{2} / 2+p_{v}^{2} / 2+\left(u^{2}+v^{2}\right) / 2$

$+\lambda u^{2} v^{2}\left(u^{2}+v^{2}\right) / 8=2 \varepsilon \quad$ where $\lambda=y^{2} /(-2 E)^{1 / 2}$ and $\varepsilon=1 /(-2 E)^{1 / 2}$. This Hamiltonian has a scaling invariance so that the classical dynamics depend only on one parameter, $\beta=\lambda \varepsilon^{2}=$ $y^{2} /(-2 E)^{3 / 2}$. Poincaré surfaces of sec-
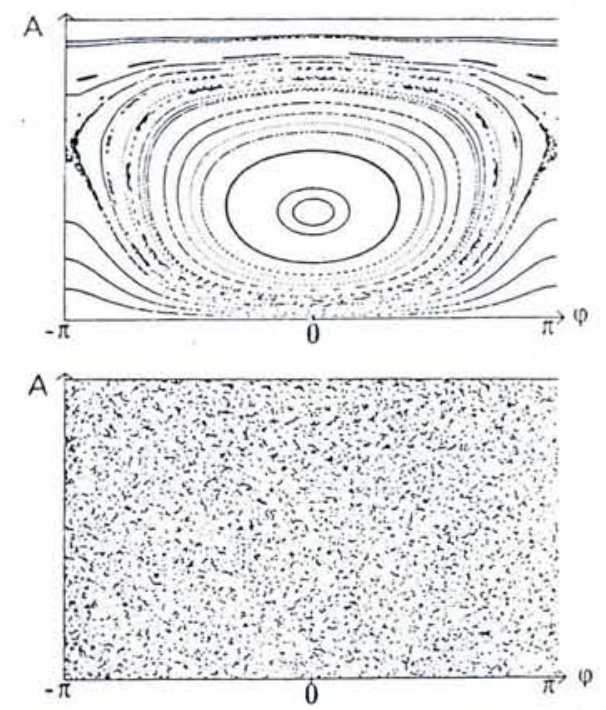

Fig. 3 - Poincaré surfaces of sections for a hydrogen atom in a magnetic field. Surfaces with $p_{y}=0$ are plotted using $E_{u}$ and $\phi$ is coordinates. Motion is nearly regular for $\beta=0.8$ (upper) and becomes fully chaotic on increasing $\beta$ to 70 (from Ref. 5).

tions for $p_{y}=0$ using $E_{u}=p^{2}+u^{2}$ versus $\phi=\arctan \left(p_{\mathrm{u}} / u\right)$ as coordinates are plotted in Fig. 3 for $\beta=0.8$ and $\beta=$ 70 (taken from Ref. 5). The transition from a nearly regular motion with a few KAM regions to fully chaotic motion is apparent. The fixed point corresponds to a circular orbit in a plane perpendicular to the magnetic field.

It should be mentioned that chaotic behaviour in Hamiltonian systems results from a transition from an integrable to a non-integrable system under perturbations which break the symmetry that leads to the integral. An integral places the trajectory on a subspace of the phase space. In non-Hamiltonian systems, the Liouville theorem is not applicable and dissipation will cause one to access a subspace of the phase space. Forced nonlinear systems with dissipation will access trajectories called strange attractors in such subspaces. Sets of nearby trajectories end up in these attractors so information about initial conditions is lost.

\section{Conclusions}

The stability properties of systems must be well understood in order to study the transition to chaos. Another important aspect is the extraction of information about a system from experimentally observed frequency data. In spite of these shortcomings, recent developments in the theory of chaos permit a better understanding of nonlinear systems. It is necessary to let some of them filter into physics curricula where undergraduate instruction in classical mechanics presently concentrates on linear, integrable systems.
Acknowledgement

The author acknowledges useful discussions with Professor Y. Nutku.

\section{REFERENCES}

[1] Gleick J., Chaos, Making a New Science (Penguin Books, New York) 1988.

[2] Lichtenberg A.J. and Lieberman M.A., Regular and Stochastic Motion (Springer Verlag, New York) 1983.

[3] Arnold V.J., Mathematical Methods of Classical Mechanics (Springer Verlag, New York) 1978; Abraham R. and Marsden J.E., Foundations of Mechanics (The Benjamin/ Cummings Publishing Co., Inc., Reading, Mass.) 1978.

[4] Cvitanovic P., Universality in Chaos (Adam Hilger Ltd, Bristol) 1984.

[5] Delande D. and Gray J.C., Phys. Rev. Lett. 57 (1986) 2006; Harada A. and Hasegawa H., J. Phys. 16A (1983) L259.

\section{Chaotic Software}

The first Physics CTI (Computers in Teaching Initiative) Newsletter and Version 1 of the Physics Courseware Catalogue were issued last September. They can be obtained by contacting the Physics CTI Centre, Department of Physics, University of Surrey, Guildford, GU2 $5 \mathrm{XH}$, UK (Tel. ++44 483 509329). The catalogue gives the availability (in the UK only) and a short description of each of nearly 70 different computer software packages for teaching physics that have been announced to the Centre. They are classified into 11 categories such as optics, quantum mechanics, etc.

Readers of our article on chaotic behaviour in dynamical systems by Professor Hacinliyan may be interested to note that the catalogue describes six packages for modelling examples of these systems, especially the simple pendulum. For instance, various effects discussed in the article such as period doubling leading to chaos for a damped, driven pendulum can be studied using a programme developed by Salford University.

The catalogue concentrates on software developed at British universities for BBC personal computers and for IBM compatibles. Future versions will describe the more commercial software, as well as the increasing number of programmes available in the US for the Mackintosh. The Centre would also like to include details of European software for teaching physics that is available in languages other than English. Information should be sent directly to the Physics CTI Centre.

\section{Research Facilities}

The combined proceedings of the four EPS Seminars on International Research Facilities provide a definitive survey of the big physics facilities in the building or planning phases. The set comprising the Zagreb (1989), London (1986), Copenhagen (1983) and Rome (1979) proceedings are available from the EPS Secretariat for 120 SFR $(60$ SFR to IOM's). Individually, they cost 60 , 65,25 and 60 SFR respectively (half price to IOM's). 